\title{
Anaphylactic shock and acute myocardial infarction following intravenous ceftazidine
}

\section{G L Punchihewa ${ }^{1}$, K R Gunatilaka ${ }^{2}$ and R Fernandopulle ${ }^{3}$}

A 72-year old hypertensive woman on nifedipine had an intracapsular fracture of the right femoral neck, following a fall. She did not give any history of food or drug allergies, or of any atopic diseases such as asthma or eczema. Her blood pressure was $150 / 90 \mathrm{mmHg}$.
Preoperative echocardiography revealed an ejection fraction of 50\%, and her chest xray and ECG were normal. Her blood urea $(8.3 \mathrm{mmol} / \mathrm{L})$, haemoglobin $(11 \mathrm{~g} / \mathrm{dL})$, fasting blood glucose $(6.4 \mathrm{mmol} / \mathrm{L})$ and serum electrolytes ( Na $138 \mathrm{mmol} / \mathrm{L}, \mathrm{K} 4.2 \mathrm{mmol} / \mathrm{L}$ ) were within normal limits.

${ }^{1}$ Orthopaedic Surgeon, National Hospital of Sri Lanka, ${ }^{2}$ Demonstrator and ${ }^{3}$ Senior Lecturer, Department of Pharmacology, Faculty of Medicine, Colombo, Sri Lanka.

Correspondence: RF, e-mail:rohinifernandopulle@hotmail.com (Competing interests: none declared). Received 16 September 2004 and revised version accepted 6 November 2004. 
Four days after admission at induction of anaesthesia, she was administered intravenous ceftazidime (1g) as surgical prophylaxis. Five to ten minutes after this she collapsed with an unrecordable blood pressure and thready pulse. She did not have difficulty in breathing, but rhonchi were heard on auscultation. This was diagnosed as anaphylactic shock and treated with promethazine $250 \mathrm{mg}$ iv, hydrocortisone $200 \mathrm{mg}$ iv, nalaxone $400 \mathrm{mg}$ iv, ephedrine $150 \mathrm{mg}$ iv, and 4 litres of isotonic saline iv. An ECG done immediately showed a left bundle branch block. She was thereafter monitored in the intensive care unit.

On day 2 after the reaction she developed macroscopic haematuria. A subsequent urine culture indicated a significant growth of Pseudomonas aeruginosa. Her renal function was normal with a serum creatinine level of $56 \mu \mathrm{mol} / \mathrm{L}$ and blood urea of $12.8 \mathrm{mmol} / \mathrm{L}$. She was treated with norfloxacin and did not develop any further reaction.

On day 5 following the reaction she had biochemical and ECG evidence of myocardial infarction. In the ward she was found to be allergic to the adhesive plasters used as dressings. She underwent surgery 3 weeks later and had an uneventful recovery.

\section{Discussion}

Correlating the timing of events with the administration of ceftazidime, a probable diagnosis of anaphylactic shock or severe hypotension following a rapid administration of ceftazidime seems likely. She had evidence of bronchospasm on auscultation, and responded to hydrocortisone and promethazine, which supports that diagnosis. Adrenaline had not been given because she was a known hypertensive, although it is stressed that adrenaline is not contraindicated in individuals with hypertension or underlying ischaemic heart disease, as the decrease in filling pressure due to anaphylaxis is likely to result in further coronary ischaemia [1] Adrenaline should be given intramuscular with careful monitoring.

Anaphylaxis is a rare adverse reaction of ceftazidime with a frequency of $0.0001-0.1 \%$ [1]. Deaths following anaphylaxis have been reported. The haematuria may have been caused by ceftazidime, as cephalosporins with the N-methylthiotetrazole (NMTT) side chain (cefamandole, cefotetan and cefoperazone) have been associated with an increased risk of bleeding due to hypoprothrombinaemia. Her prothrombin time had not been assessed.

The myocardial infarction is likely to have resulted from the anaphylactic episode as it occurred immediately after administration of ceftazidime. Allergy induced acute coronary syndromes have been reported with the beta lactams [2]. There are a variety of methods for causality assessment of adverse drug reaction reports.

According to a widely used scoring method [3] our case report could be classified as a probable adverse reaction to ceftazidime. Hypotensive reactions to intravenous cephalosporins could have been be avoided by slow administration. A previous history of severe or immediate anaphylaxis to cephalosporins is a contraindication.

We conclude that the probable diagnosis in this patient is anaphylactic shock leading to a myocardial infarction following the administration of ceftazidime. Based on current evidence, we emphasise that the benefit of using appropriate doses of intramuscular adrenaline in anaphylactic reactions involving respiratory difficulty or hypotension, far exceeds the risk in patients with ischaemic heart disease.

\section{Acknowledgements}

We thank Dr. A Ranatunga, Medical Officer, National Hospital of Sri Lanka for reporting this case to the drug information centre.

\section{Reference}

1. Pramod S Kelkar, James T-C Li. Cephalosporin allergy. New England Journal of Medicine 2001; 345: 804-9.

2. Moreno-Ancillo Á, Domínguez-Noche C, Gil-Adrados A C, Cosmes Martn P M. Acute coronary syndrome due to amoxicillin allergy. Allergy 2004; 59: 466-7.

3. Naranjo CA, Busto U, Sellers EM, Sandor P, Ruiz I, et al. A method for estimating the probability of adverse drug reactions. Clinical Pharmacology and Therapeutics 1981; 30: 239-45. 